\title{
Temporal Patterns in Student-Advisor Instant Messaging Exchanges: Individual Variation and Accommodation
}

\author{
Amaury de Siqueira \\ Indiana University, Bloomington \\ adesique@indiana.edu
}

\author{
Susan C. Herring \\ Indiana University, Bloomington \\ herring@indiana.edu
}

\begin{abstract}
This study analyzed rhythm and timing in 18 instant messenger (IM) conversations between one advisor and four graduate students (4 dyads), hypothesizing that individuals would show a consistent style across sessions but would differ from one another across $d y$ ads. ANOVA results supported the hypotheses for individual students and dyads; however, the advisor varied the timing of her messages to harmonize with the students' preferred temporal styles. These findings suggest that individuals' temporal consistency with themselves may be disrupted if they accommodate to others; advisors may be especially likely to do this. A correlation was also found between number of characters and seconds per message across sessions, on the basis of which we posit an overall temporal profile for dyadic IM. Implications of the findings are discussed for online advising, instruction, and system design.
\end{abstract}

\section{Introduction}

Conversations tend to have a rhythm; messages are exchanged at a particular rate, with pauses of a moreor-less consistent length between turns. At the same time, speakers in face-to-face conversations vary in their preference for faster or slower turn transitionsfor example, according to their cultural backgrounds [32]. Differing temporal expectations can result in misunderstanding and conversational breakdown, with speakers with faster and slower styles finding the interaction frustrating and/or negatively judging each other's character and intentions [33].

It seems plausible that individuals would also differ in their interactional styles when communicating via computer-mediated communication (CMC). Such variability could have consequences for the success of computer-mediated interactions, as has been previously observed for face-to-face conversations. Temporality has been addressed to a limited extent in CMC research, e.g., in studies of synchronicity [36] and normative email response rates $[20,21]$. However, to our knowledge, no studies have considered individual tim- ing preferences and how these mesh-or not-in interactive CMC.

This study analyzed individuals' and dyads' temporal signatures (rhythm and message production timing) in spontaneously-occurring instant messenger (IM) conversations. The exchanges took place between one North American university professor and four of her graduate student advisees, each of whom was from a different culture (four dyads). We expected that each individual would show a consistent temporal style across sessions within his or her dyad, but would differ from each other across dyads, and that a similar pattern would be evident for dyads. Results from one-way and two-way ANOVA showed this to be the case for both individuals and dyads - they were consistent with themselves but differed from one another-with one interesting exception. The professor varied the timing of her messages to harmonize with individual students' preferred temporal styles and, as a result, was inconsistent with herself. A correlation was also found between number of characters and seconds across sessions, on the basis of which we posit an overall temporal profile for dyadic IM.

These findings provide evidence that IM users have individual temporal styles, yet may accommodate to others' preferred styles, within the overall constraints of synchronous IM. The concept of accommodation helps explain why individuals with different styles are able to communicate successfully online. It also raises questions about the circumstances under which such accommodation occurs - was the professor more likely to accommodate because of her gender and/or her advisory role? - and the effects of technical factors such as system synchronicity on accommodation. In concluding, we discuss the implications of these findings for online advising, instruction, and system design.

\section{Background}

\subsection{Chronemics}

Chronemics is the study of nonverbal cue systems and communicators' perceptions of, and reactions to, 
time [35]. Such phenomena have been investigated extensively in spoken communication, including in clinical contexts $[7,22]$. In an early study of chronemics in CMC, Burgoon and Saine [6] manipulated the time stamps of email messages to create an artificial sense that work-related emails were sent after hours and that personal emails were sent during the time the sender would normally be expected to be at work. The temporal manipulations seemed to have positively influenced users' satisfaction and impression formation.

Walther and Tidwell [36] later manipulated time stamps on asynchronous email messages to assess variations in the interpretation of dominance, affection, and intimacy related to temporal factors. The researchers predicted that time variations would affect all three variables, and the findings from the study partially supported their predictions. Messages were perceived as more dominant if they were sent at night and were task-oriented. Perceived affection resulted from the combination of time sent, content, and promptness of reply. Messages were considered the most affectionate if they were a quick reply, sent during the morning, and related to a task message. Slow replies were considered to show moderate affection, regardless of the time of the day. Extreme delays in replies corresponded to the lowest measurements of affection.

Kalman et al. [21] examined response delays in several asynchronous CMC corpora, including public discussion forums and the Enron email corpus. They found a linear pattern of response latency distributions and identified three CMC latency zones: quick, above average, and long silence. The majority of responses (roughly $80 \%$ ) fell within the 'quick zone;' only a negligible minority of responses fell in the 'long silence' zone. These results were highly stable across the observed groups; the authors claimed that a similar distribution characterizes synchronous CMC and face-toface conversation.

Bays [2, 3] suggested that temporal patterns in synchronous Internet Relay Chat are a function of complex visual-verbal interactions. She proposed the existence of a beat and tempo rhythm in IRC interactions, despite the fact that they take place via typed text. Perceived temporal patterns are cognitively processed and associated in the minds of participants with a set of social meanings and expectations. According to Bays [3], the desire to sustain a perceived rhythm on screen is a factor motivating participants to take predictable linguistic turns in synchronous online interactions.

The early CMC chronemics studies cited above explored the effects of temporal manipulations on users' sense of satisfaction and impression formation [6, 36]. While the results are suggestive of possible links between chronemic factors and individuals' interaction with the medium, these studies relied on experimental methods, and the results may not generalize to spontaneous communication. A newer generation of chronemics researchers has studied aspects and possible meanings of temporal patterns in naturally-occurring asynchronous and synchronous CMC [2, 20, 21]. In both approaches, however, the role of the individual has been secondary to an attempt to generalize about patterns of rhythm and overall group effects.

Results from these studies highlight the importance of chronemics in the use of CMC systems. However, it is clear that the $\mathrm{CMC}$ literature on chronemics has not yet adequately addressed temporal effects at the level of individual differences.

\subsection{Accommodation}

Accommodation theory originated as a sociopsychological model of speech-style modifications, based on the observation that speakers often adapt their messages to take account of the characteristics of their listeners [12]. Modifications may occur at any linguistic level, including language complexity (as when people simplify their language when speaking to babies or foreigners [10]), politeness [5], accent [11], and choice of language [13]. Especially relevant to the present study, speakers have also been found to accommodate to one another in extra-linguistic behaviors such as utterance length [24], speech rate [31], pause length [18], and response latency [8].

Accommodation is generally considered to have beneficial effects. Giles, Taylor, and Bourhis [13] found that in the bilingual context of Montreal, the more effort a speaker was perceived to have made toward convergence in language choice (French vs. English), the more favorably that person was evaluated and the more the other speaker converged in return. According to Giles, Coupland, and Coupland [12], accommodative processes can also facilitate language learners' proficiency in a foreign language, influence job satisfaction, and affect satisfaction with medical encounters. Foreign students who accommodate to the pragmatic norms of their host culture also have more successful advising sessions with faculty [1].

Few studies have examined accommodation in CMC. Bunz and Campbell [5] studied politeness accommodation in email and found that the most polite messages received the most polite responses, showing accommodation. However, an earlier study by Myers [27] found that "leaders" maintained their styles in postings to an electronic bulletin board system, supporting maintenance rather than accommodation. This study points to the important fact that accommodation is sensitive to status, with lower-status individuals (or 
members of lower-status groups) tending to accommodate to higher-status individuals more than the inverse.

Accommodation is also sensitive to gender. In a study of males and females in various dyads, Bilous and Krauss [4] found that females converged toward males in number of words uttered. More generally, women tend to be more addressee-oriented in their interpersonal communication than men [17]. This would seem to support the principle that the less socially-statusful group (women) accommodates to the more socially-statusful group (men). In mixed-sex dyads, however, Mulac et al. [26] found that both genders adopted a linguistic style more like that of their outgroup partner than they would have maintained with an in-group partner. This is similar to Herring's [15] finding that the numerically less-represented gender in two asynchronous academic discussion lists tended to accommodate to the linguistic style of the majority gender on the list. Both studies report cross-gender accommodation that involves men accommodating to women as well as women accommodating to men.

The above research demonstrated that speakers may accommodate to one another in terms of timing, that accommodation is sensitive to status and gender, and that accommodation may occur at the group level in CMC. However, no studies, to our knowledge, have investigated individual accommodation as regards the timing of messages in any mode of CMC.

\subsection{Academic advising}

Faculty play an important socializing role in students' college experience [23]. Academic advising is often the primary occasion for direct contact between a student and a faculty member. Yarbrough [37] suggests that it is important to clarify the individual roles and responsibilities of the student-advisee and the professor-mentor relationship to enhance student selfefficacy for completing the degree requirements. Bardovi-Harlig and Hartford [1] emphasize the importance of respecting institutional hierarchy in formal advising encounters.

Advising can also take place informally, and be equally, if not more, beneficial. Research has found that more frequent informal encounters with faculty can significantly increase students' persistence in their academic programs, especially when the encounters focus on intellectual or course-related matters [30]. Informal advising has been claimed to be more effective than formal contact in increasing student's creative development [23]. Gaff, cited in [23], posits that the single largest difference between influential faculty and their colleagues is the extent to which interaction occurs outside the classroom. Faculty who interact most often with students outside the classroom tend to be highly engaged with teaching, irrespective of gender and rank [23].

Computer-mediated communication provides abundant opportunities for students and faculty to interact informally. Moreover, it is often claimed that textbased CMC has an inherently "democratizing" effect, leveling the status of participants [cf. 14]. As academic advising is increasingly carried out via email, chat, social network sites-or, as in the case of the present study, instant messaging - the possibility arises that institutional hierarchy will break down. However, the effects of informal advising via CMC on accommodation patterns have yet to be investigated, to our knowledge.

\section{Methodology}

\subsection{Data collection}

The data for this study are typed conversations that took place using Skype's instant messaging application. Skype is a service that enables users to communicate with one or more users via a proprietary Voiceover-Internet-Protocol (VoIP). Skype IM is a one-way synchronous text-based communication tool that enables two or more personal computer users to communicate in real-time using text as a medium. Messages can be logged for future viewing or immediately deleted from the server once the application is closed.

A Skype IM log contains four kinds of information: a) date stamp; b) time stamp; c) user's identity; d) message unit. The data source can be exported as a raw file in ASCII format containing alphanumeric characters and invisible text delimiters (i.e., tab, character return, and paragraph markers). Once created, the raw file can be imported and formatted in a statistical software package or spreadsheet application.

The logs for the current study were generated by four dyads, comprised of four doctoral students (two male and two female) and one female professor, their academic advisor. The conversations, all but one of which were initiated by the students, sought the advice of the professor on academic matters and took place spontaneously over a period of several months. All participants were experienced IM users, and the students had used IM to communicate with the professor previously. The students had different linguistic backgrounds (Bengali, English, Portuguese, Russian), but all were fluent speakers and writers of English who had lived and studied in the United States for two or more years. All five participants shared a professional interest in computer-mediated communication. Specific topics discussed in the sessions included university requirements, the logistics of ongoing research, and plans for future projects. 
Participants were asked to volunteer their IM logs for the purpose of this study. We aimed to collect five complete IM sessions from each of the four dyads. Because of problems with data access, however, only three IM sessions were available for the native English-speaking student, bringing the total number of sessions ("conversations") to 18 .

\subsection{Data analysis}

Raw data from the IM logs containing date stamp, time stamp, user ID, and message unit were formatted and imported into a spreadsheet program, and later, into a statistical software package, for analysis. Values were calculated for the following measures:

Number of words (words) in each message. A single space character was used as the operational delimiter of a word. For example, "ruthere?" was considered to be one word, while "are you there?" contains three words.

Number of characters (char) in each message. This value comprises all keystrokes, including space, tabs, and return keys. Thus the message "I am good" contains 10 characters - seven alphanumerical characters, two spaces, and one character return (in order to submit the message).

Seconds per message (secs). This was calculated by subtracting the value for the time stamp of a message from the time stamp value of a previous message. Results were reported in seconds (e.g., one minute and 20 seconds was represented as 80 seconds). The first message of every chat record received no value.

Production time (prtim), indicates the overall time a user takes to produce a message, from the second a new message appears on the screen to the moment the user hits the enter key to submit a reply to the message. Unlike typing speed, production time value comprises typing speed plus internal/external factors. Internal factors include cognitive processing time, reading/writing proficiency, and facility with the technology; external factors include interruptions, parallel task processing not related to the topic (multitasking), initial messages, and cut and pasted text. The following categories that can affect production time were identified and coded:

Initial message. The first message of each chat record was often followed by a delay before the intended addressee, the professor, saw the initiation and responded. This is because she routinely turned off the sound on her computer and thus did not receive an auditory signal indicating that a message had arrived.

Disrupted adjacency. A message that does not logically relate to the immediately preceding message (cf. Herring, 1999), but rather replies to an ear- lier message, and for which the typing plausibly started before the immediately preceding message, can give rise to (the appearance of) an improbably short production time.

External interference. External factors can cause the production time of messages to be delayed or shortened. A message containing, e.g., 'brb' (be right back) followed by a long pause suggests an interruption due to external factors. This category includes instances when users' engage in parallel processing of tasks not related to the content of interaction (multitasking). It also includes long cutand-pasted strings of characters that artificially lower production time values.

Cognitive. This code indicates that an extreme production time value is plausibly the result of internal cognitive processes related to tasks associated with the topic of interaction. For example, a long pause following a message asking "how many speech acts do you see?" suggests cognitive processing during which the addressee is counting units before submitting a response.

Messages exhibiting extreme low and high production time values as a result of response delays to initial messages, disrupted adjacency, or external interferences (i.e., physical absence, parallel task processing, cut and paste) were excluded from computation. However, extreme values generated as a result of cognitive processing of tasks directly related to the interaction were included in the analysis, as they were part of the individual variation the study was designed to investigate.

Log values for characters, seconds, and production time (logchar, logsecs, logprtim). These values represent the log conversion for all values reported on number of characters, seconds, and production time. Logarithmic conversion was required in order to address the skewness of the original values. Descriptive, one-way, and two-way analyses of variance (ANOVA) were conducted based on the log conversion values.

The above values were calculated separately for the advisor and for each student. In addition, the couple (or dyad) was analyzed as a composite unit made up of the advisor and one student. The term dyad sample refers to all IM sessions between the advisor and one student.

\section{Research question and hypotheses}

The overall question addressed by this study is:

RQ: To what extent is the production time of text messages in student-advisor IM chat consistent, as opposed to variable?

Determining whether there is a correlation between characters and production time is a necessary first step 
towards determining the predictability (or stability) of production time measurements. While it seems selfevident that longer character strings have longer production times, this may not be the case if internal factors (e.g., cognitive effort, facility with the technology) vary greatly across individuals. Moreover, it is important to establish any timing constraints that are characteristic of IM exchanges at the outset, in order to understand what is and is not possible using this particular technology. Thus we first hypothesized:

H1: Messages with more characters will have a longer production time, regardless of who typed them or when. Specifically:

H1a: There will be a positive overall correlation between number of characters produced and seconds.

H1b: The correlation between seconds and characters will not be significantly different across all IM sessions.

The second hypothesis concerns the advisor, who occupies a role distinct from that of the students and is the only person in our data to participate in more than one dyad. We hypothesized that:

H2: The advisor's production time will show less variation across IM sessions than across dyads. Specifically:

H2a: The advisor's production time will not be significantly different across IM sessions within a dyad sample.

H2b: The advisor's production time will be significantly different across dyad samples. It follows, therefore, that:

H2c: The advisor's production time variation within IM sessions will be less than her production time variation across dyad samples.

The third hypothesis concerns the students' production times. Analogous to $\mathrm{H} 2$ above, we hypothesized that:

H3: Students' production time will show significantly less variations across IM sessions than across dyad samples. Specifically:

H3a: Students' production time will not be significantly different across IM sessions.

H3b: Students' production time will be significantly different across dyad samples. It follows, therefore, that:

H3c: Students' production time variation within IM sessions will be less than students' production time variation across dyad samples.
The last set of hypotheses concerns dyads, considered as a single unit, without respect to differences between advisor and student.

H4: Dyads' production times will show less variation across IM sessions than across dyad samples.

H4a: Dyads' production times will not be significantly different across IM sessions.

H4b: Dyads' production time will be significantly different across dyad samples.

H4c: The variability of dyads' production time across IM sessions will be less than the variability of production time across dyad samples.

\section{Results}

\subsection{Overall production time}

Hypothesis 1a predicted that there would be a positive overall correlation between number of characters produced per message and seconds per message. A regression of 'LOGCHAR' on 'LOGSECS' with a covariate for ID shows significant correlation at $\mathrm{F}$ $(1,17)=.000$ at $p<.5$ (see Table 1$)$. These results support H1a.

Dependent Variable LOGSECS
\begin{tabular}{|l|c|c|c|}
\hline \multicolumn{1}{|c|}{ Source } & $\mathrm{df}$ & $\mathrm{F}$ & Sig. \\
\hline LOGCHAR & 1 & 407.514 & .000 \\
\hline DATE & 17 & 5.569 & .000 \\
\hline
\end{tabular}

a. R Squared $=.489$ (Adjusted R Squared $=.475$ )

Table 1. Participants' overall production time

$\mathrm{H} 1 \mathrm{~b}$ predicted that the correlation between seconds and characters per message would not be significantly different across IM sessions. Two-way ANOVA shows significant interaction between DATE and LOGCHAR. That is, the correlation between LOGCHAR and LOGSECS is significantly different, $F$ $(1,17)=.001, \mathrm{p}<.01$, across IM sessions (Table 2). These results contradict $\mathrm{H} 1 \mathrm{~b}$.

Dependent Variable LOGSECS

\begin{tabular}{|l|c|c|c|}
\hline \multicolumn{1}{|c|}{ Source } & $\mathrm{df}$ & $\mathrm{F}$ & Sig. \\
\hline LOGCHAR & 1 & 235.616 & .000 \\
\hline DATE & 17 & 2.592 & .000 \\
\hline DATE* LOGCHAR & 17 & 2.436 & .001 \\
\hline
\end{tabular}

a. $R$ Squared $=.538$ (Adjusted $R$ Squared $=.495$ )

Table 2. Participants' production time across IM sessions 


\subsection{Advisor's production time}

Hypothesis 2a predicted that the advisor's production time would not be significantly different across IM sessions within a dyad sample. A two-way ANOVA on LOGPRTIM with DATE and COUPLE (dyad) as independent variables shows that advisor's production time is not significantly different across dates (sessions) $\mathrm{F}(1,3)=.302, p<.5$, after adjusting for COUPLE (see Table 3). These results support H2a.

Dependent Variable LOGPRTIM
\begin{tabular}{|l|c|c|c|}
\hline \multicolumn{1}{|c|}{ Source } & $\mathrm{df}$ & $\mathrm{F}$ & Sig. \\
\hline COUPLE & 3 & 1.577 & .195 \\
\hline DATE(COUPLE) & 14 & 1.162 & .302 \\
\hline
\end{tabular}

a. $\mathrm{R}$ Squared $=.538$ (Adjusted $\mathrm{R}$ Squared $=.495$ )

Table 3. Advisor's production time across IM sessions

Hypothesis $2 \mathrm{~b}$ predicted that the advisor's production time would be significantly different across dyad samples. A one-way ANOVA on LOG of PRTIM with COUPLE (dyad) as independent variable indeed shows significant differences across couples $\mathrm{F}(1,3)=.018$, $p<.5$ (Table 4). These results support $\mathrm{H} 2 \mathrm{~b}$; that is, the advisor's timing varies depending on the student with whom she is interacting.

Dependent Variable LOGPRTIM

\begin{tabular}{|c|c|c|c|}
\hline Source & $\mathrm{df}$ & $\mathrm{F}$ & Sig. \\
\hline COUPLE & 3 & 3.384 & .018 \\
\hline
\end{tabular}

a. R Squared $=.026$ (Adjusted R Squared $=.018$ )

Table 4. Advisor's production time across dyads

Hypothesis 2c states that the advisor's production time variation within IM sessions will be less than her production time variation across dyad samples. Since the results from $\mathrm{H} 2$ a show no significant difference in production time across IM sessions within dyads, and the results from $\mathrm{H} 2 \mathrm{~b}$ show significant differences across dyads, these two findings taken together support H2c.

\subsection{Students' production time}

Analogous to hypothesis 2a, hypothesis 3a predicted that students' production time would not be significantly different across IM sessions. A two-way ANOVA on LOG PRODTIM with DATE and ID as independent variables shows no significant differences across dates $\mathrm{F}(1,17)=.100, p<.5$, after adjusting for Student (see Table 5).

Dependent Variable LOGSECS
\begin{tabular}{|l|c|c|c|}
\hline Source & $\mathrm{df}$ & $\mathrm{F}$ & Sig. \\
\hline DATE(ID) & 14 & 1.520 & .100 \\
\hline ID & 3 & 4.187 & .006 \\
\hline
\end{tabular}

a. R Squared $=.098$ (Adjusted R Squared $=.062$ )

Table 5. Students' production time across IM sessions

Hypothesis $3 \mathrm{~b}$ asserts that students' production time will be significantly different across dyad samples. A one-way ANOVA on LOG PRODTIM with ID as independent variable shows significant differences among students with $\mathrm{F}(1,3)=.000, p<.001$ (Table 6). These results support $\mathrm{H} 3 \mathrm{~b}$, demonstrating that student production time is significantly different across dyad samples.

Dependent Variable LOGSECS
\begin{tabular}{|l|c|c|c|}
\hline Source & $\mathrm{df}$ & $\mathrm{F}$ & Sig. \\
\hline ID & 3 & 8.279 & .000 \\
\hline
\end{tabular}

a. $R$ Squared $=.053$ (Adjusted $R$ Squared $=.047$ )

Table 6. Students' production time across dyads

Hypothesis $3 \mathrm{c}$ states that students' production time variation across IM sessions within a dyad will be less than their production time variation across dyad samples. Since the results from H3a show no significant difference in production across IM sessions, and the results from $\mathrm{H} 3 \mathrm{~b}$ show significant differences across dyads, these two findings taken together support $\mathrm{H} 3 \mathrm{c}$.

\subsection{Dyads' production time}

Hypothesis 4 concerns the production times of dyads, units made up of the advisor and one student.

H4a states that dyads' production time will not be different across IM sessions within a dyad. A two-way ANOVA on LOG PRODTIM with DATE and COUPLE as independent variables shows no significant difference across dates, with $\mathrm{F}(1,14)=.418, p<.5$ (Table 7), hence $\mathrm{H} 4 \mathrm{a}$ is supported.

Dependent Variable LOGPRTIM
\begin{tabular}{|l|c|c|c|}
\hline \multicolumn{1}{|c|}{ Source } & $\mathrm{df}$ & $\mathrm{F}$ & Sig. \\
\hline DATE(COUPLE) & 14 & 1.032 & .418 \\
\hline COUPLE & 3 & 5.197 & .001 \\
\hline
\end{tabular}

a. $\mathrm{R}$ Squared $=.055$ (Adjusted R Squared $=.036$ )

Table 7. Dyad's production time across IM sessions

Hypothesis $4 \mathrm{~b}$ states that dyads' production time will be significantly different across dyad samples, that is, that dyads will differ from each other. A one-way ANOVA on LOG PRODTIM with COUPLE as independent variable shows significant differences across 
dyads, with a $\mathrm{F}(1,3)=.000, p<.001$ (Table 8). These results support $\mathrm{H} 4 \mathrm{~b}$.

Dependent Variable LOGPRTIM
\begin{tabular}{|c|c|c|c|}
\hline Source & $\mathrm{df}$ & $\mathrm{F}$ & Sig. \\
\hline COUPLE & 3 & 11.041 & .000 \\
\hline
\end{tabular}

a. R Squared $=.039$ (Adjusted R Squared $=.035$ )

Table 8. Dyad's production time across dyad samples

Finally, hypothesis $4 \mathrm{c}$ predicted that the variability in dyads' production time across IM sessions would be less than the variability in production times across dyad samples. Given that the results from H4a show no significant difference for dyads in overall production time across IM sessions, and that the results from $\mathrm{H} 4 \mathrm{~b}$ show a significant difference across dyads, it follows that $\mathrm{H} 4 \mathrm{c}$ is also supported.

\section{Discussion}

The results for the first set of hypotheses showed that the number of characters in the IM messages examined was positively correlated with production time overall, as hypothesized. However, variation was found across sessions as regards rate of character production. This is important to establish; both commonalities and variation call for explanation.

We note again that the overall correlation is not an indication of similarity in typing speed. Characters and seconds were calculated per message; the correlation means that messages tended to be produced at a constant ratio, regardless of the typing speed of the individual users. Some users favored short, quick messages, and others favored long messages sent less often. The advisor sent messages representing all increments along the dimension of short/fast to long/slow, depending on which student she was interacting with.

This similar overall ratio may reflect the temporal constraints of IM, as distinct from other CMC modes. It may also indicate accommodation, a norm that these IM users unconsciously converge towards in order to co-construct a harmonious rhythm. Interesting evidence in support of the latter speculation can be seen in the exchanges of one student, Alvaro, with the advisor, Laura. (All names are pseudonyms.) Alvaro is a slow typist; he compensates by sending frequent, short messages. Laura's messages to Alvaro are typed more quickly but are longer, with the result that the average time each person takes to produce a message is similar. In this way, even though their typing speeds differ, the members of this dyad achieve a convergent rhythm.

The variation within individuals across sessions suggests that message production time can be affected by the nature of the task and/or the topic of the interac- tion. The purpose of another student, Anil's, sessions with Laura, for example, varied considerably-one session was initiated to request specific help with data analysis, another was to solicit general feedback on a presentation, and yet another was to discuss arrangements for a social gathering - and the timing of his messages was also variable. Laura's timing also varied across sessions, because her data included sessions from all four dyads.

The results for hypotheses 2 and 3 support the idea that individuals are consistent with themselves across IM sessions, and that these internally-consistent temporal styles vary across individuals. The second part of this result is perhaps not surprising, given that each student is from a different culture (although the variation might also be due to individual differences; our study design and small sample cannot decide this question). The point is that these IM users manifest individual temporal styles, giving rise to the possibility of style conflict and the undesirable consequences that could entail.

In these data, that possibility is avoided, due primarily to the efforts of the advisor, who varies her message timing to harmonize with that of each student. The advisor, like the students, tended to be consistent with herself across sessions within each dyad, but she was (necessarily, since the students differed across dyads, and she accommodated to each) inconsistent with herself across dyads. The fact that the dyads exhibited the same behavior as the individuals, as shown in the results for hypothesis 4, lends further support to the claim that accommodation was taking place within dyads. The advisor, when asked, affirmed that her accommodating behavior was entirely unconscious; this is supported by the fact the conversations took place before the idea for this study arose.

It remains to explain the extent and directionality of accommodation in these IM interactions. The dyads in this study did not meet in the middle; if they had, we would expect to see greater similarity across dyads. The significant differences across dyads support the view that it was the advisor who converged towards the students more than the inverse. This requires explanation, since accommodation theory predicts that members of lower status groups (such as students) should accommodate to members of higher status groups (such as professors). However, [1] found that international graduate students did not always accommodate (e.g., show deference) to their faculty advisors appropriately; rather, they sometimes argued with their advisors' recommendations and issued bald requests, as if they and the advisors were status equals. The fact that three of the four students in the present study were international students may have led them not to accommodate, leaving the advisor to do so instead. 
It is also possible that Laura, as a senior female professor, adopted a nurturing stance toward her advisees, all of whom she knew well and was on friendly terms with, that included interactional accommodation. It is an open question whether a male professor would be equally accommodating in a similar situation; the present data allow us only to speculate that gender and advisor role make a difference.

\section{Conclusions}

\subsection{Summary}

The findings of this study lead to the conclusion that IM interactions are governed by underlying temporal patterns, much as email-based discussion forums were found to exhibit a normative pattern of response rates [21]. Evidence of an overall rhythmic flow in IM dyadic interactions was presented. Previous work did not take account of individual variation; this study found that IM patterns were consistent within individuals but significantly dissimilar across individuals.

At the same time, each IM user operates within production time constraints that are dependent on task, circumstances, and personal time limitations, potentially giving rise to variation across sessions. In this study, such variation was found to be less than the variation among different individuals. Thus the study provides robust evidence of the existence of individual temporal styles in instant messaging. This is a significant finding that suggests the importance of future research into temporal style preferences in online communication and their potential influence in instruction.

The findings of this study further suggest that users accommodate to each other during extended mutual interaction and that temporal style differs across cultures. Since the study was not designed to address these questions directly, these results are not conclusive. However, unconscious accommodation toward the individual rhythm and timing preferences of each student did occur in this instance, demonstrating that such accommodation can take place via IM, much as it does in face-to-face communication [8, 31].

\subsection{Implications}

The implications of the existence of a preferred temporal pattern in synchronous online interactions are far reaching. Previous research on face-to-face interaction has suggested that individuals prefer to communicate by exchanging turns in a rhythm that resembles their own. Speakers with different preferred rhythms may not only cause others to feel uncomfortable; their different styles may make them targets of negative judgments, as in the case of New York Jewish speakers who were judged by Californians to be pushy because of their tendency to overlap turns with a previous speaker [33]. (Conversely, the New Yorkers judged the Californians to be dull and disengaged, because they paused before taking a next turn [33].) Temporal accommodation in such cross-cultural contexts, online and offline, serves important social functions.

With international students, accommodation plays a complex role. On the one hand, native speakers typically accommodate to foreigners, e.g., by slowing their speech rate and articulating distinctly [10]; instructors do this as well. On the other hand, there is often an expectation that institution hierarchy should be respected, such that students of all cultural backgrounds are expected to accommodate to faculty members, and may be considered pragmatically inappropriate if they do not [1]. In deciding whether the use of synchronous CMC is appropriate with certain students, faculty advisors should be aware of the potential of such tools to encourage informal, egalitarian interaction. While this may not always be desirable, it could benefit international students by relieving them of the pragmatic burden of manifesting deference (including through temporal accommodation), allowing them to focus on other goals in advising sessions. More generally, advisors should be aware that individual students may have different temporal styles and that accommodation may be necessary to ensure successful interaction.

The findings of this study may help instructors and school administrators in the process of adopting CMC technology to implement blended and full online courses. Previous studies have reported inconsistent results regarding learners' self-reported satisfaction when using CMC tools. Some studies have found students to be significantly less satisfied with computermediated learning [28, 29], while other studies [9, 19] found students to be more satisfied with computermediated than with face-to-face collaborative processes. It is possible that differences in students' preferred temporal cadence in CMC interactions may account for some of the inconsistency in this literature.

Awareness of learners' preferred temporal styles could also inform instructional strategies and prepare learners and instructors to better understand participation in online discussions. For example, instructors could introduce online ice-breaker activities in order to familiarize students with each other's preferred temporal styles. An orientation session foregrounding temporal differences prior to online collaborative activities could also enhance tolerance among team members, while controlling for learners' temporal expectations.

Finally, individual differences could assist instructional designers in determining what CMC technology to use, given particular kinds of users, and in designing activities that are sensitive to temporal preferences. 
The findings from this study could provide guidance especially where the design of online instruction requires incorporating communication tools to support collaborative work. They suggest that decisions regarding the deployment of synchronous and asynchronous $\mathrm{CMC}$ tools in the design of online instruction should go beyond considering the requirements of task typology, time constraints, and available technology, to take account of learners' temporal styles.

\subsection{Limitations and future research}

This is a preliminary study of temporal style in online communication. The small size of the sample18 IM conversations produced by five users-limits the generalizability of the study's findings. Moreover, this convenience sample did not allow for systematic testing of possible effects of participant demographics such as gender, age, and nationality. Future studies should analyze larger, systematically-selected samples. Two potentially fruitful venues for future research would be to examine temporal patterns in relation to gender and cultural differences.

A second limitation is the nature of crossparticipant interaction in our data, which restricts the generalizability of claims about accommodation, since only one user's data (the professor's) could be compared across dyads. Ideally, data would be analyzed from all study participants interacting with each other.

The tendency to accommodate plausibly depends on variables such as an interlocutor's role (e.g., student/teacher, employer/employee, professional/client), social status, gender, and/or culture. In the present study, these variables generated conflicting predictions (e.g., the professor is higher status and should maintain her style; the professor is female and should accommodate). These conflicts should be explored and clarified in future studies that investigate larger populations and/or control for participant demographics.

In the present study, the advisor and the students were on friendly terms before the data were collected. It is possible that a pattern of advisor-to-student accommodation had already been established before the online interaction took place, rather than occurring as a result of the online environment. Research is needed that compares accommodation in student-instructor interactions in offline and online environments, in order to understand fully the effects of technological mediation on advising. Moreover, this study examined only academic IM conversations. The academic context might have influenced the rhythm and patterns of communication, which might not generalize to other institutional or recreational contexts of IM use. These should be analyzed in their own right.
Finally, we only investigated conversations that took place using IM. At the same time, the finding of an overall temporal profile for the IM conversations is intriguing. Applying similar methods to other modes of computer-mediated interaction could validate or disprove the claim that overall patterns of message response in online interactions remain the same, even as absolute timings differ [21]. Given that the synchronicity of a medium has previously been claimed to cause significant variation in interaction patterns [35], it would be interesting, indeed, if some temporal aspects of mediated communication proved to be constant.

\section{References}

[1] Bardovi-Harlig, K., \& Hartford, B. S. (1990). Congruence in native and nonnative conversations: Status balance in the academic advising session. Language Learning, 40(4), 467501 .

[2] Bays, H. La politesse sur Internet: le don des objets imaginaires: Enquêtes de terrain et analyses de corpus. Colloque, Louvain-la-Neuve, Belgium, 107(2000), 169-183. http://cat.inist.fr/?aModele $=$ afficheN\&cpsidt=13539603.

[3] Bays, H. (1998), Framing and face in Internet exchanges: A socio-cognitive approach. LinguistikOnline, 1. http://www. linguistik-online.de/bays.htm.

[4] Bilous, F., \& Krauss, R. (1988). Dominance and accommodation in the conversational behaviors of same- and mixed-gender dyads. Language and Communication, 8, 183-194.

[5] Bunz, U., \& Campbell, S. W. (2004). Politeness accommodation in electronic mail. Communication Research Reports, 21(1), 11-25.

[6] Burgoon, J. K., \& Saine, T. (1978). The unspoken dialogue: An introduction to nonverbal communication. Boston: Houghton Mifflin Company.

[7] Bruneau, T. (1973). Communicative silences: Forms and functions. Journal of Communication, 23, 17-46.

[8] Capella, J. N., \& Planalp, S. (1981). Talk and silence sequences in informal conversations III. Interspeaker influence. Human Communication Research, 7, 117-132.

[9] Cohen, A., \& Scardamalia, M. (1998). Discourse about ideas monitoring and regulation in face-to-face and computer-mediated environments. Interactive Learning Environments, 6(1-2), 93-113.

[10] Ferguson, C. (1971). Absence of copula \& the notion of simplicity: A study of normal speech, baby talk, foreigner talk, \& pidgins. In D. Hymes (Ed.), Pidginization and creolization of languages (pp. 141-151). Cambridge, UK: Cambridge University Press. 
[11] Giles, H. (1973). Accent mobility: A model and some data. Anthropological Linguistics 15, 87-105.

[12] Giles, H., Coupland, J., \& Coupland, N., Eds. (1991). Contexts of accommodation: Developments in applied sociolinguistics. Cambridge, UK: Cambridge University Press.

[13] Giles, H., Taylor, D. M., \& Bourhis, R. Y. (1973). Towards a theory of interpersonal accommodation through language use: Some Canadian data. Language in Society, 2, $177-$ 192.

[14] Herring, S. C. (1993). Gender and democracy in computer-mediated communication. Electronic Journal of Communication, 3 (2). http://ella.slis.indiana.edu/ herring/ejc.txt.

[15] Herring, S. C. (1996). Two variants of an electronic message schema. In S. C. Herring (Ed.), Computer-mediated communication: Linguistic, social and cross-cultural perspectives (pp. 81-108). Amsterdam: John Benjamins.

[16] Herring, S. C. (1999). Interactional coherence in CMC. Journal of Computer-Mediated Communication, 4(1). http://jcmc.indiana.edu/vol4/issue1/herring.html.

[17] Holmes, J. (1990). Hedges and boosters in women's and men's speech. Language and Communication, 10(3), 185205.

[18] Jaffe, J., \& Feldstein, S. (1970). Rhythms and dialogue. New York: Academic Press.

[19] Jonassen, D. H., \& Kwon, H. (2001). Communication patterns in computer-mediated versus face-to-face group problem solving. Educational Technology Research and Development, 49(1), 35-51.

[20] Kalman, Y. M., \& Rafaeli, S. (2005). Email chronemics: Unobtrusive profiling of response times. Proceedings of the 38th Hawaii International Conference on System Sciences. Los Alamitos, CA: IEEE Press.

[21] Kalman, Y. M., Ravid, G., Raban, D., \& Rafaeli, S. (2006). Pauses and response latencies: A chronemic analysis of asynchronous CMC. Journal of Computer-Mediated Communication, 12(1). http://jcmc.indiana.edu/vol12/issue1/ kalman.html.

[22] Kruger, H-P. (1989). Speech chronemics-A hidden dimension of speech: Theoretical background, measurement and clinical validity. Pharmacopsychiatry, 22, 5-11.

[23] Lamport, M. A. (1993). Student-faculty informal interaction and the effect on college student outcomes: A review of the literature. Adolescence, 28, 971-990.

[24] Matarazzo, J. D., Wiens, A. N., Matarazzo, R. G., \& Saslow, G. (1968). Speech and silence behavior in clinical psychotherapy and its laboratory correlates. In J. M. Shlien (Ed.), Research in Psychotherapy. Vol. III (pp. 347-394). Washington, D.C,: American Psychological Association.
[25] Meijden, H., \& Veenman, S. (2005). Face-to-face versus computer-mediated communication in a primary school setting. Computers in Human Behavior, 21, 831-859.

[26] Mulac, A., Wiemann, J. M., Widenmann, S. J., \& Gibson, T. W. (1988). Male/female language differences and effects in same-sex and mixed-sex dyads: The gender-linked language effect. Communication Monographs, 55, 315-335.

[27] Myers, D. (1987). 'Anonymity is part of the magic': Individual manipulation of computer-mediated communication contexts. Qualitative Sociology, 10(3), 251-266.

[28] Ocker, R. J., \& Yaverbaum, G. J. (2001). Collaborative learning environments: Exploring student attitudes and satisfaction in face-to-face and asynchronous computer conferencing settings. Journal of Interactive Learning Research, 12(4), 427-448.

[29] Olaniran, B. A., Savage, G. T., \& Sorenson, R. L. (1996). Experimental and experiential approaches to teaching face-to-face and computer-mediated discussion. Communication Education, 45(3), 244-259.

[30] Pascarella, E. T., \& Terenzini, P. T. (1977). Patterns of student-faculty informal interaction beyond the classroom and voluntary freshman attrition. The Journal of Higher Education, 48(5), 540-552.

[31] Street, R. L., JR. (1983). Non content speech convergence and divergence in adult-child interaction. In $\mathrm{R}$. Bostrom (Ed.), Communication Yearbook 7 (pp. 369-395). Beverly Hills, CA: Sage.

[32] Tannen, D. (2000). Don't just sit there-interrupt! Pacing and pausing in conversational style. American Speech, 75(4), 393-395.

[33] Tannen, D. (1985). Silence - anything but. In D. Tannen \& M. Saville-Troike (Eds.), Perspectives on silence (pp. 93111). Norwood, NJ: Ablex.

[34] Thakerar, J. N., Giles, H., \& Cheshire, J. (1982). Psychological and linguistic parameters of speech accommodation theory. In C. Fraser \& K. R. Sherer (Eds.), Advances in the social psychology of language (pp. 205-255). Cambridge, UK: Cambridge University Press.

[35] Walther, J. B. (2002). Time effects in computermediated groups: Past, present, and future. In P. Hinds \& S. Kiesler (Eds.), Distributed work (pp. 235-257). Cambridge, MA: MIT Press.

[36] Walther, J. B., \& Tidwell, L. C. (1995). Nonverbal cues in computer-mediated communication, and the effect of chronemics on relational communication. Journal of Organizational Computing, 5, 355-378.

[37] Yarbrough, D. (2002). The engagement model for effective academic advising with undergraduate college students and student organizations. Journal of Humanistic Counseling, Education and Development, 41, 61-68. 